Check for updates

Cite this: RSC Adv., 2017, 7, 42320

Received 17th June 2017

Accepted 21st August 2017

DOI: $10.1039 / c 7 r a 06759 c$

rsc.li/rsc-advances

\title{
Synergistic effects of zwitterionic segments and a silane coupling agent on zwitterionic shape memory polyurethanes $\uparrow$
}

\author{
Zaochuan Ge, Huanhuan Ren, Shuqin Fu* and Shaojun Chen (D) *
}

\begin{abstract}
Recently, zwitterionic shape memory polyurethanes (ZSMPUs) have found many promising applications in smart biomedical field. This paper provides a novel strategy to improve shape memory properties of ZSMPUs by using a silane coupling agent, namely 3-glycidyloxypropyl-trimethoxysilane (KH560). The synergistic effects of zwitterionic segments and silane coupling agents on the structure, morphology, thermal properties, mechanical properties, and shape memory properties were carefully investigated. The results demonstrate that the incorporation of $\mathrm{KH} 560$ does not influence the original molecular structure and morphology of ZSMPUs, and the KH560-modified ZSMPUs form a hard-soft phase separation structure. Also, the KH560 modification promotes the entanglement of hard segments, and thus provides better mechanical properties to ZSMPUs-KH560. Moreover, the silane coupling agent can provide lubrication to influence the aggregation of soft segments while the zwitterionic segments promote the formation of crystal seeds. Therefore, a good semi-crystalline soft phase endows the ZSMPUs-KH560 with a decent shape fixity (e.g. above 94\%), and the entangled hard segments greatly enhance the shape recovery of ZSMPUs (e.g. above 90\%).
\end{abstract}

\section{Introduction}

Shape memory polyurethanes (SMPs) are widely studied for scientific and technological applications because of their wellknown responsiveness to specific external stimuli and great ability to remember their original shape. ${ }^{1-3}$ Until now, various stimuli, such as light, heat, moisture, and $\mathrm{pH}$ value, have been used to adjust the macroscopic properties of SMPs. ${ }^{4-6}$ In comparison with shape memory alloys and shape memory ceramics, SMPs have advantages of light-weight, good biocompatibility, high deformability, and easy adjustment of shape recovery temperature. ${ }^{7-9}$ However, some performance defects, such as low stiffness and weak recovery stress, accompany many SMPs. Thus, great efforts have been made to overcome these drawbacks in the past decades. ${ }^{\mathbf{5 , 1 0}}$

An important strategy to deal with these shortcomings is the hybridization of SMPs with fillers such as particles, fibers, and tubes. ${ }^{11-13}$ SMPs composites are being rapidly developed to address the need for mechanical reinforcement in smart practical applications. ${ }^{\mathbf{1 3 - 1 5}}$ Previous research work also indicates that

Guangdong Research Center for Interfacial Engineering of Functional Materials, Shenzhen Key Laboratory of Polymer Science and Technology, Shenzhen Key Laboratory of Special Functional Materials, Nanshan District Key Lab for Biopolymers and Safety Evaluation, College of Materials Science and Engineering, Shenzhen University, Shenzhen 518060,China.E-mail: chensj@szu.edu.cn; gezc@ szu.edu.cn; Fax: +86-755-26534562; Tel: +86-755-26534562

† Electronic supplementary information (ESI) available. See DOI: 10.1039/c7ra06759c the polymers modified via hybridization method usually have higher strength, better stiffness, and some special characteristics which can offer further advantages over SMPs. ${ }^{16,17}$ In general, chemical hybridization is better than physical blending due to fine dispersion and improved interfaces between polymers and fillers. ${ }^{\mathbf{1 8}, 19}$ The fillers modified by chemical hybridization can achieve multifunctional crosslinking with polymers, thereby enhancing the rubber elasticity and strain recovery. ${ }^{20}$ Among the various chemical hybridization agents, silane coupling agents with general formula of $\mathrm{R}-\left(\mathrm{CH}_{2}\right)_{n}-\mathrm{Si}-\left(\mathrm{OR}^{\prime}\right)_{3}$ are widely used to modify fillers or polymers. ${ }^{21}$ Several literature works have reported that the silane coupling agents generate bridging with the surface of fillers, and greatly enhance the physical bonding between the fillers and the polymer matrix. Therefore, modification technologies based on silane coupling agents have drawn significant attention from researchers. For instance, Yan et al. have reported one kind of polyurethane composite incorporated with nano-Cu fillers which was firstly modified with 3-aminopropyltriethoxy silane before usage. ${ }^{22} \mathrm{Li}$ et al. used 3-glycidyloxypropyltrimethoxysilane (KH560) to improve the interfacial interaction between nano- $\mathrm{CaCO}_{3}$ and the polyurethane matrix. ${ }^{23}$ Zhang et al. dispersed KH560 in epoxy resin and found that modification effect can be optimized by adjusting the dosage of silane coupling agent. ${ }^{24}$ Additionally, Li et al. introduced KH560 to increase cohesion with polyacrylate and reduce the residue of pressure-sensitive adhesives without sacrificing the tack or peeling strength. ${ }^{25}$ These studies confirm that silane coupling agents, particularly KH560, are 
great chemical hybridization agents for the modification of various polymers. However, until now, only a few studies have focused on the modification of SMP systems through the incorporation of silane coupling agents. The modification with KH560 might open new avenues in the development of multifunctional SMPs.

Recently, we developed a novel kind of shape memory polyurethanes (SMPUs), namely zwitterionic shape memory polyurethanes (ZSMPUs), by integrating both anionic and cationic groups in the same segment. ${ }^{26-28}$ The incorporation of zwitterionic segments greatly enhanced the biocompatibility and antibacterial activity of ZSMPUs. ${ }^{29}$ However, the zwitterionic segments ruined the strain recovery in both $N$-methyldiethanolamine (MDEA) based ZSMPUs $^{27}$ and $N, N$-bis(2hydroxylethethyl) isonicotinamide (BINA) based ZSMPUs. ${ }^{26}$ To promote the promising applications of ZSMPUs in smart biomedical fields, their capability of shape fixation and shape recovery should be urgently improved. In another paper, we reported about the synthesis of biocompatible ZSMPUs consisting of zwitterionic hard segments based on MDEA and 1,3propanesultone (1,3-PS) and polyethylene glycol (PEG) soft segments. The preliminary results show that the strain recovery is massively ruined by the MDEA-PS segments. As a follow-up to this work, herein we plan to modify the MDEA based ZSMPUs via chemical hybridization method by using KH560. Hence, in this work, we present our insights into the influence of MDEAPS zwitterionic hard segments on KH560-modified ZSMPUs (referred as ZSMPUs-KH560). Thus, in this paper, we meticulously investigated the synergistic effects of zwitterionic segments and silane coupling agents on the structure, morphology, thermal properties, mechanical properties, and shape memory properties of ZSMPUs-KH560.

\section{Materials and methods}

\subsection{Materials}

The raw materials used in this study include dibutyltin dilaurate (DBTDL, analytical reagent), 1,6-hexamethylene diisocyanate (HDI, analytical reagent), dimethylformamide (DMF, analytical reagent), polyethylene glycol (number-average molecular weight 6000, PEG6000, premium grade), $N$-methyldiethanolamine (MDEA, analytical reagent),1,3-propanesultone (1,3-PS, analytical reagent), and 3-glycidyloxypropyltrimethoxysilane (KH560, analytical reagent). All these raw materials were purchased from Aladdin Chemical Reagent Co. Ltd. (Shanghai, China) and used without further purification.

\subsection{Preparation of ZSMPUs-KH560}

As shown in Table 1, ZSMPUs-KH560 were synthesized with various zwitterionic segment contents based on different molar ratio of 1,3-PS and MDEA. The reaction routes for the synthesis of ZSMPUs-KH560 are presented in Scheme 1. The raw materials including MDEA, DMF, DBTDL, and HDI were added in order at $60{ }^{\circ} \mathrm{C}$. After vigorous mechanical stirring at $80{ }^{\circ} \mathrm{C}$ for $1.5 \mathrm{~h}$, PEG6000 was added to the mixture and the reaction system was stirred for another $4 \mathrm{~h}$. After the synthesis of prepolymer of MDEA-PEG-HDI polyurethane system, 1,3-PS was added and the ring-open reaction was conducted hermetically at $75{ }^{\circ} \mathrm{C}$ for $24 \mathrm{~h}$. The resultant ZSMPU samples were coded as SMPU-\$PS, where $\$$ is the molar ratio of 1,3-PS and MDEA, such as $0.2,0.4,0.6,0.8$, or 1.0. After the synthesis of ZSMPUs, the temperature of the reaction system was kept at $140{ }^{\circ} \mathrm{C}$; the silane coupling agent KH560 was added to the ZSMPUs system via another ring-open reaction for $2 \mathrm{~h}$. The final product was obtained after evaporation of DMF from the resultant ZSMPUsKH560/DMF solution in a Teflon pan at $80^{\circ} \mathrm{C}$ for $8 \mathrm{~h}$ in vacuum. The samples of the target ZSMPUs-KH560 were collectively coded as SMPU-\#PS-KH560, where \# is the molar ratio of 1,3-PS and MDEA, such as $0.2,0.4,0.6,0.8$, or 1.0.

\subsection{Instrumentation and measurements}

The molecular structure of the target ZSMPUs-KH560 was analyzed by Fourier transform infrared (FT-IR) spectrometry recorded in the range of $4000-400 \mathrm{~cm}^{-1}$. The test samples were mechanically compressed and scanned using a Nicolet FT-IR (Thermo Nicolet FTIR 6700, Nicolet, America).

The crystallization behavior of the target ZSMPUs-KH560 was analyzed with X-ray diffractometry (XRD, D8 Advance, Bruker, Germany). The crystalline morphology was identified by a polarized optical microscope (POM, MP41, Mshot, China).

The morphological characterization of the target SMPUs was performed using atomic force microscopy (AFM, Nanonavi ESweep, SII Nanotechnology Instrument, Japan) in tapping mode and scanning electron microscopy (SEM, NGB4-DXS10AC, Hitachi, Japan).

The impact of KH560 on the crystallization temperature $\left(T_{\mathrm{c}}\right)$ and crystal melting temperature $\left(T_{\mathrm{m}}\right)$ of the target ZSMPUs-

Table 1 Composition of ZSMPUs-KH560

\begin{tabular}{|c|c|c|c|c|c|c|c|}
\hline \multirow[b]{2}{*}{ Samples } & \multirow{2}{*}{$\begin{array}{l}\text { Soft segment }(\mathrm{g}) \\
\text { PEG6000 }\end{array}$} & \multicolumn{2}{|c|}{ Hard segment $(\mathrm{g})$} & \multirow[b]{2}{*}{$1,3-\mathrm{PS}(\mathrm{g})$} & \multirow[b]{2}{*}{ KH560 (g) } & \multirow[b]{2}{*}{$n$ (PS/MDEA) } & \multirow[b]{2}{*}{$m$ (KH560/HDI) } \\
\hline & & HDI & MDEA & & & & \\
\hline SMPU-0.4PS-KH560 & 11.67 & 8.01 & 3.32 & 1.36 & 0.2816 & $0.4: 1$ & $0.2: 1$ \\
\hline SMPU-0.6PS-KH560 & 11.67 & 8.01 & 3.32 & 2.04 & 0.2816 & $0.6: 1$ & $0.2: 1$ \\
\hline SMPU-0.8PS-KH560 & 11.67 & 8.01 & 3.32 & 2.72 & 0.2816 & $0.8: 1$ & $0.2: 1$ \\
\hline
\end{tabular}






Scheme 1 Synthesis route of synthesis of ZSMPUs-KH560.

KH560 was studied by differential scanning calorimetry (DSC, Q200, TA universal, America) in nitrogen.

Dynamic mechanical analysis (DMA, Q200, TA universal, America) of the target ZSMPUs-KH560 with approximate dimensions of $18 \mathrm{~mm} \times 6 \mathrm{~mm} \times 0.4 \mathrm{~mm}$ was conducted in nitrogen in the temperature range of $-50{ }^{\circ} \mathrm{C}$ to $180{ }^{\circ} \mathrm{C}$.

The tensile mechanical properties were investigated using Instron 4465 testing machine on the rectangular $(20 \mathrm{~mm} \times$ $4 \mathrm{~mm} \times 0.5 \mathrm{~mm})$ specimens at the elongation rate of $10 \mathrm{~mm} \mathrm{~min}^{-1}$ and the strain-stress curves were recorded for analysis. All the measurements of the target ZSMPUs-KH560 discussed above were repeated three times.

\section{Results and discussion}

\subsection{Structural analysis}

Fig. 1 shows typical FT-IR spectra obtained from ZSMPUs and ZSMPUs-KH560. Structure comparison of KH560, SMPU-0.2 PS, and SMPU-0.2PS-KH560 was performed using FT-IR (see Fig. 1A). The as-prepared sample SMPU-0.2 PS shows a peak attributed to $\mathrm{N}-\mathrm{H}$ stretching vibration at $3333 \mathrm{~cm}^{-1}$ and a peak attributed to $\mathrm{C}=\mathrm{O}$ stretching vibration at $1697 \mathrm{~cm}^{-1}$, which confirms the formation of urethane group and stronger hydrogen bonding in hard segments. Additionally, there is stretch vibration frequency at $1035 \mathrm{~cm}^{-1}$ and strong vibration frequency at $955 \mathrm{~cm}^{-1}$ in both SMPU-0.2 PS and SMPU-0.2PS$\mathrm{KH} 560$, which can be ascribed to the existence of $\mathrm{S}=\mathrm{O}$ group and quaternary $\mathrm{N}$-group, respectively. As expected, no significant differences were observed between SMPU-0.2 PS and SMPU-0.2PS-KH560 (see Fig. 1A). Hence, the zwitterionic structure is confirmed in both ZSMPUs and ZSMPUs-KH560, and the incorporation of KH560 does not affect the original zwitterionic structure and hydrogen bonding within zwitterionic polyurethanes. In addition, Fig. 1B demonstrates that in all samples of ZSMPUs-KH560, the intensity of $\mathrm{S}=\mathrm{O}$ peak enhanced with the increase of the molar ratio of 1,3-PS and MDEA. Also, the addition of KH560 in SMPU-0.2PS-KH560 resulted in novel absorption bands at $1079 \mathrm{~cm}^{-1}$ and $1049 \mathrm{~cm}^{-1}$, which are assigned to $\mathrm{Si}-\mathrm{O}$ stretch vibration and $\mathrm{Si}-$ O-C stretch vibration, respectively. These peaks were overlapped by the absorption of $S=O$ group of sulfonic acid group at approximately $1035 \mathrm{~cm}^{-1}$. However, the absorption peak at approximately $1253 \mathrm{~cm}^{-1}$ and $909 \mathrm{~cm}^{-1}$, corresponding to the stretching vibration of the epoxy group in KH560, disappeared completely in ZSMPUs-KH560. These results illustrate that KH560 was grafted successfully onto the primary chains of ZSMPUs-KH560. Therefore, we can safely conclude that the designed samples of ZSMPUs-KH560 were successfully prepared, and KH560 does not destroy the molecular structure of ZSMPUs in this study.

\subsection{Thermal properties}

Fig. 2 shows the DSC curves of the target ZSMPUs-KH560. The cooling DSC curves demonstrate that all ZSMPUs-KH560 polymers show crystallization transition at $c a .16 .2{ }^{\circ} \mathrm{C}$ while the 

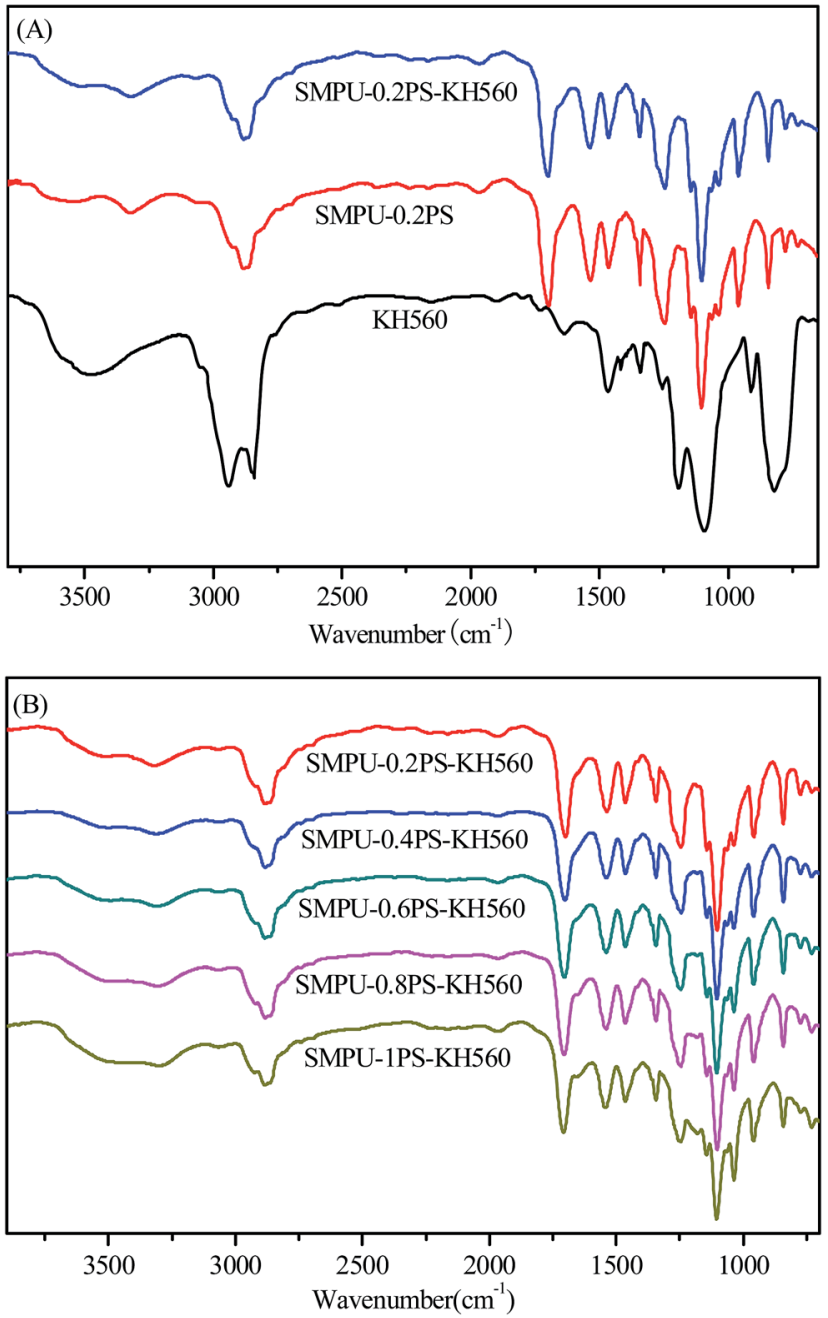

Fig. 1 FT-IR spectra showing the structure of ZSMPUs-KH560 ((A) for comparison of KH560, SMPU-0.2 PS and SMPU-0.2PS-KH560, and (B) ZSMPUs-KH560 with various zwitterionic segments).

crystal melting temperature $\left(T_{\mathrm{m}}\right)$ occurs at about $53{ }^{\circ} \mathrm{C}$ on the heating curves. This crystallization behavior could be ascribed to the presence of PEG6000 soft segment, indicating the formation of semi-crystalline soft phase. The crystallization temperature $\left(T_{\mathrm{c}}\right)$ increases from $16.2{ }^{\circ} \mathrm{C}$ (e.g. in sample SMPU0.2PS-KH560) to $24.2{ }^{\circ} \mathrm{C}$ (e.g. in sample SMPU-1.0PS-KH560) with the increase in contents of zwitterionic segment (see Fig. 2A). These results suggest that the zwitterionic segments promote the crystallization of soft segments in ZSMPUs-KH560. In comparison with the untreated ZSMPUs (see ESI $\dagger$ ), the incorporation of KH560 reduces the $T_{\mathrm{c}}$ of ZSMPUs-KH560. The possible reason for this phenomenon could be that KH560 reduces the density of nucleation sites on the surface of nucleation sites or in other words, elevate the activation energy required for nucleation of crystallites. In addition, Fig. $2 \mathrm{~B}$ also shows that the $T_{\mathrm{m}}$ shifts to a slightly lower temperature as the zwitterionic segment contents increases in the ZSMPUs-KH560. In the sample SMPU-1.0PS-KH560, the broad crystalline peak might be a result of multiple crystals, which in turn could have resulted from different zwitterionic seeds. In contrast, the $T_{\mathrm{m}}$
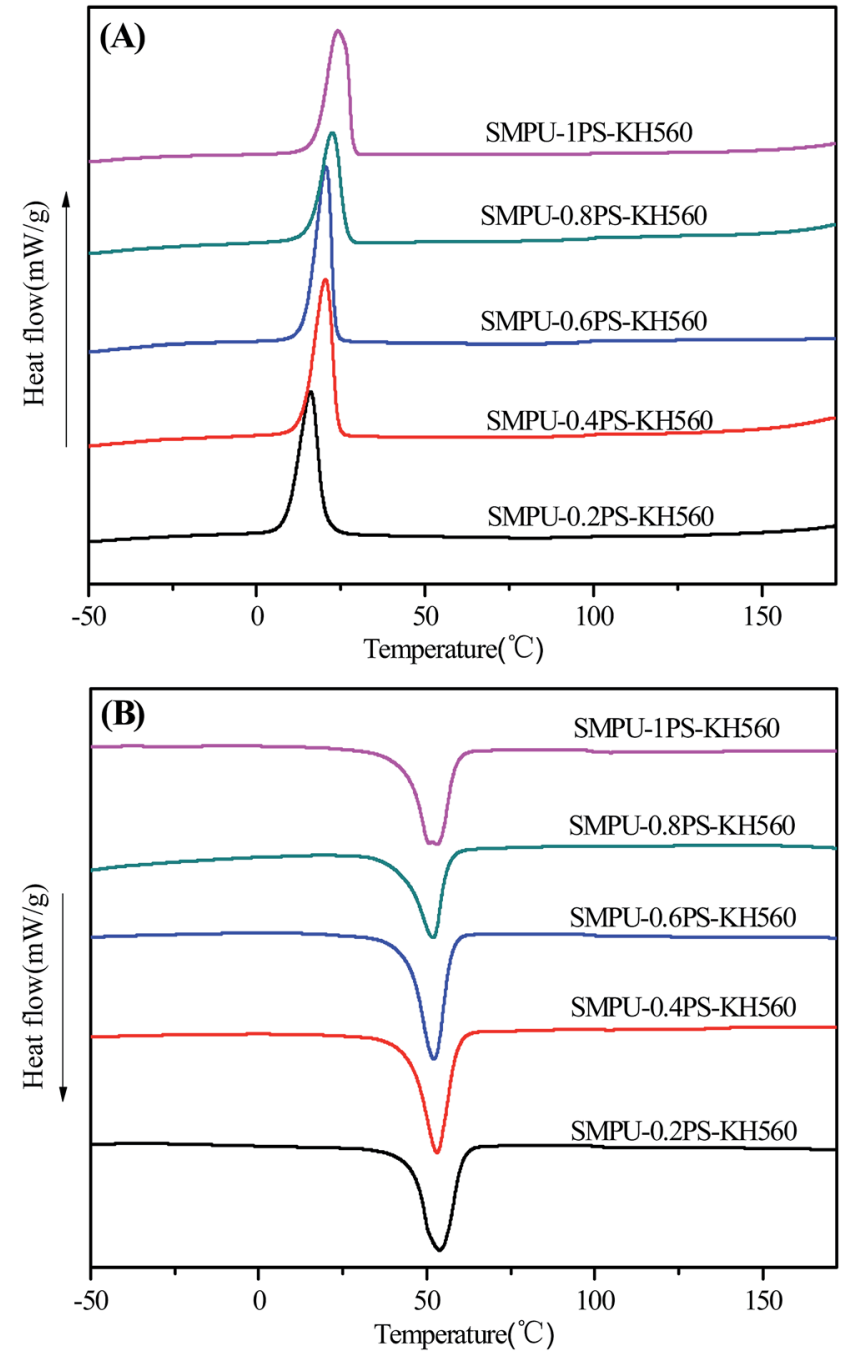

Fig. 2 The DSC curves of ZSMPUs-KH560: (A) the cooling DSC curves; and (B) the second DSC heating curves.

shifts to a higher temperature range as the zwitterionic segment contents increase in the untreated ZSMPUs. These results further confirm that the silane coupling agents act as lubrication agents to influence the aggregation of soft segments while the zwitterionic segments promote the formation of crystal seeds.

\subsection{Morphology analysis}

The morphology of ZSMPUs-KH560 was further investigated by XRD, POM, AFM, and SEM. The XRD patterns (see Fig. 3) establish that ZSMPUs-KH560 have two clear crystallization peaks near $2 \theta=19.3^{\circ}$ and $2 \theta=23.4^{\circ}$, which confirms the formation of PEG crystalline soft phase. The POM images provide a direct proof of the crystallization of PEG6000 soft segments. Fig. 4 shows that there is a "+" polarizing optical pattern of spherical crystals which is consistent with the analysis in Section 3.2. Thus it can be firmly concluded that the incorporation of KH560 onto the main chain of ZSMPUs-KH560 has no measurable impact on the crystallization of PEG6000 


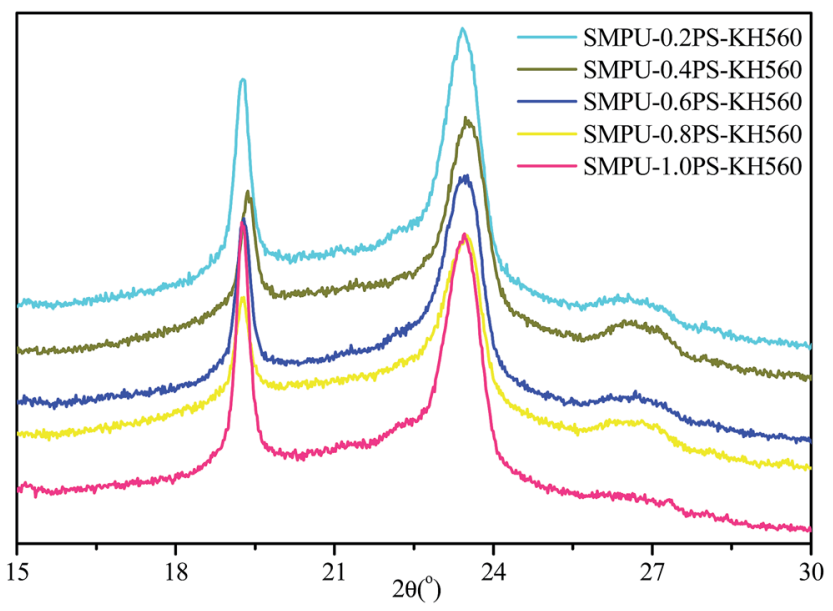

Fig. 3 XRD curves of ZSMPUs-KH560.

soft segments. Additionally, the AFM images clearly show the phase-separation structure in ZSMPUs-KH560. The cuspidal outshoots in Fig. 5 could be a result of the aggregates of HDIMDEA segments serving as hard phase, while the potholes are associated with the PEG6000 soft phase. This aggregation of zwitterions is consistent with previous investigations.

In addition, the surface morphology of ZSMPUs-KH560 was systematically investigated in this study. Fig. 6 presents the SEM images of ZSMPUs-KH560 with various contents of zwitterionic segments. The surface morphology of SMPUs-0.2PS-KH560 and SMPUs-0.4PS-KH560 contains a rough fractured surface with irregular bending wave patterns. Moreover, the fractured surface spreads randomly. This fracture appearance might have resulted from the ductile fracture which illustrates their high toughness properties. As the molar ratio of 1,3-PS and MDEA increases, an increasing number of bending waves are observed in samples SMPU-0.6PS-KH560 and SMPU-0.8PS-KH560, suggesting an enhanced ductility. In contrast, the SEM images of untreated ZSMPUs demonstrate that only the ZSMPUs with higher content of zwitterionic segments show the features of ductile fractures. These results thus confirm that the ZSMPUsKH560 possess a typical ductile morphology. The possible reason for the phenomenon could be that the integration of KH560 segments and zwitterionic segments promote the phase mixing of soft phase and hard phase since KH560 provides flexible side chains for entanglement and the ionic bonding of zwitterionic segments improve the intermolecular forces. ${ }^{30,31}$

\subsection{Tensile properties}

Fig. 7 shows the stress-strain curves of ZSMPUs-KH560 at room temperature. As presented in Fig. 7A, the elongation at break in the sample SMPU-0.2 PS was much higher than that in the sample SMPU-0.2PS-KH560, while the maximum tensile stress of ZSMPUs-KH560 was much higher. This result thus confirms that the entanglement of coupling agent greatly improves the stress, while the lubrication of KH560 significantly reduces the strain. Additionally, the stress-strain patterns (see Fig. 7B) demonstrate that with an increase in the molar ratio of 1,3-PS and MDEA, the maximum tensile stress and elongation at break decreased monotonically. ${ }^{19}$ The maximum tensile stress for samples SMPU-0.2PS-KH560, SMPU-0.4PS-KH560, SMPU0.6PS-KH560, SMPU-0.8PS-KH560, and SMPU-1PS-KH560 were $4.5 \mathrm{MPa}$, 4.3 MPa, 3.8 MPa, $3.2 \mathrm{MPa}$, and $2.3 \mathrm{MPa}$, respectively, while the elongation at break for samples SMPU0.2PS-KH560, SMPU-0.4PS-KH560, SMPU-0.6PS-KH560, SMPU-0.8PS-KH560, and SMPU-1PS-KH560 were $34.25 \%$, $19.91 \%, 33.49 \%, 21.00 \%$, and $20.36 \%$, respectively. These results suggest that the incorporation of zwitterionic segments disturb the orientation of polymer chains at high elongations. Additionally, as mentioned above, an increased content of zwitterionic segments might promote the miscibility of soft phase and hard phase, leading to a fall in the flexibility of ZSMPUs-KH560. Therefore, integration of KH560 could compensate for the negative influences of zwitterionic segments, providing better mechanical properties to ZSMPUsKH560.
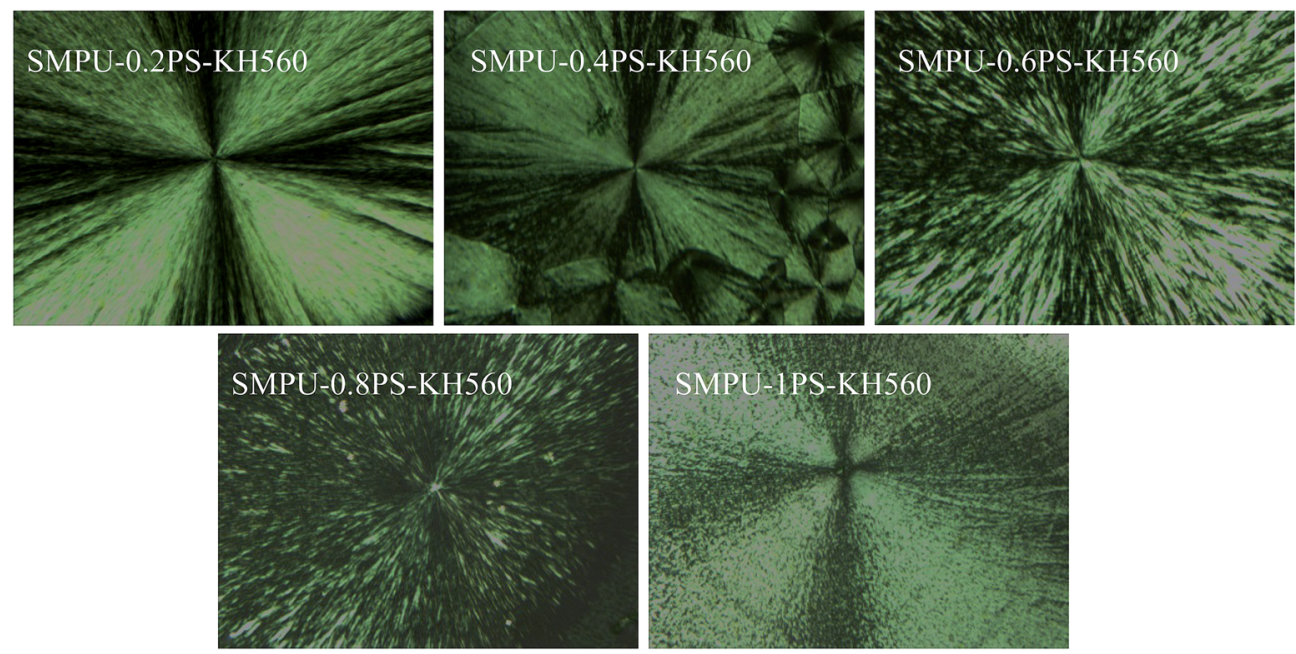

Fig. 4 POM images of ZSMPUs-KH560 (magnified 100 times). 

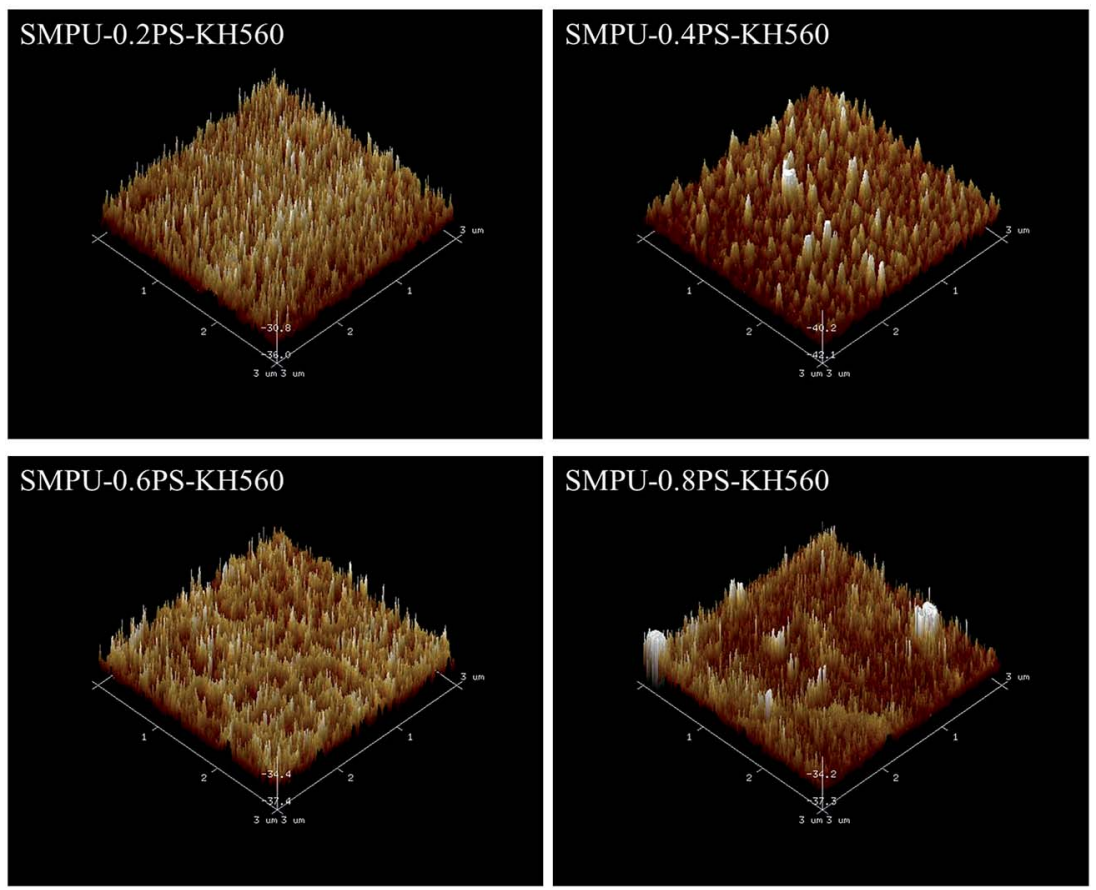

Fig. 5 AFM images for ZSMPUs-KH560.
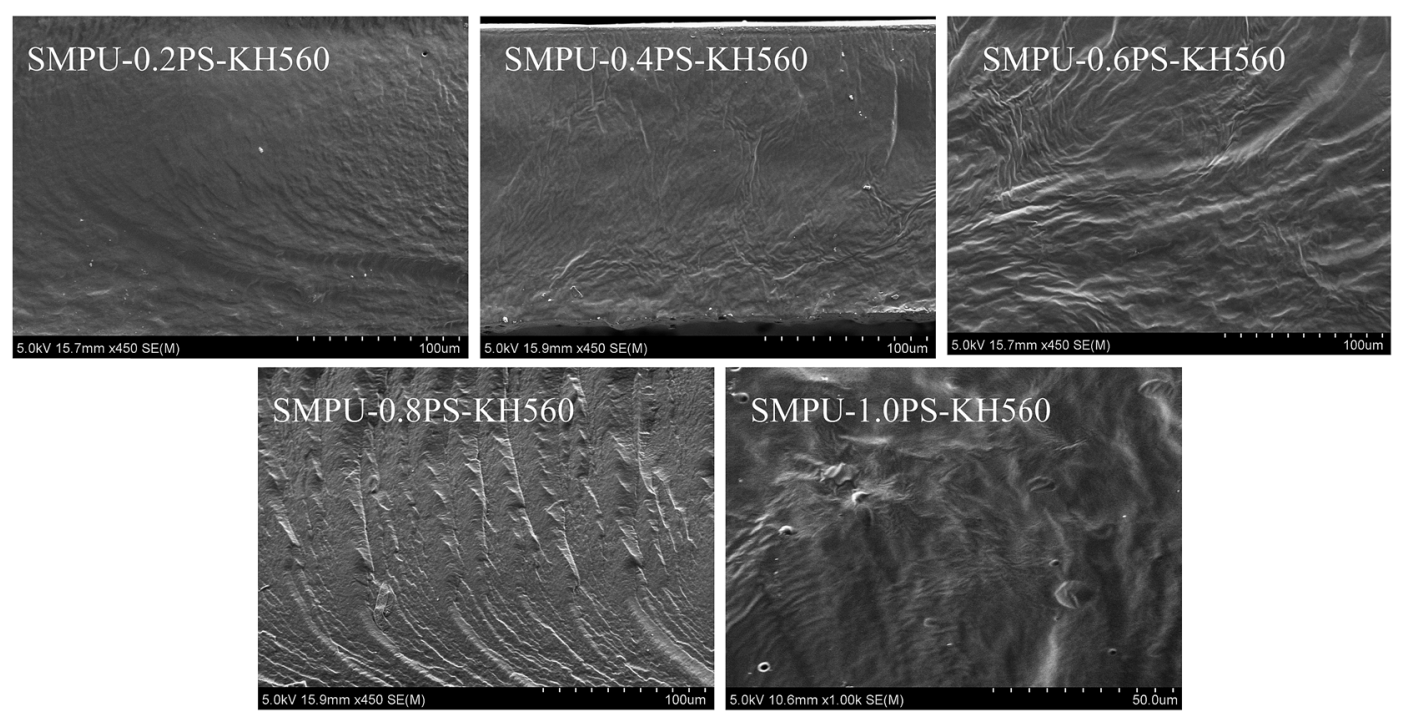

Fig. 6 SEM images of ZSMPUs-KH560.

\subsection{Dynamic mechanical properties}

The thermo-mechanical properties of the target polyurethanes were further investigated via dynamic mechanical analysis. Fig. 8A shows that all ZSMPUs-KH560 possessed extremely high storage modulus $\left(E^{\prime}\right)$ at the glassy state below $-11^{\circ} \mathrm{C}$. During the glass transition process, the $E^{\prime}$ dropped by twenty times into a semi-crystalline state. As the temperature increased further, a second significant modulus decrease occurred in the temperature range of $50-65{ }^{\circ} \mathrm{C}$, which precisely corresponds to the crystal melting temperature of ZSMPUs-KH560. Finally, the storage modulus remained stable until the temperature of
$200{ }^{\circ} \mathrm{C}$. There was no clear difference in modulus at the glassy state or the semi-crystalline state among ZSMPUs-KH560 with different content of zwitterionic segments. The storage modulus at first rose with the increase in the content of zwitterionic segments, whereas the sample SMPU-1.0PS-KH560 showed the least rubber modulus, probably due to the residues of 1,3-PS. $\tan \delta$ curves in Fig. 8B further display the increase of $T_{\mathrm{g}}$ with increase in zwitterionic segment contents, in other words from sample SMPU-0.2PS-KH560 to SMPU-0.8PS$\mathrm{KH} 560$, while the possible residues of 1,3-PS in the samples SMPU-0.4PS-KH560 and SMPU-1.0PS-KH560 result in the 

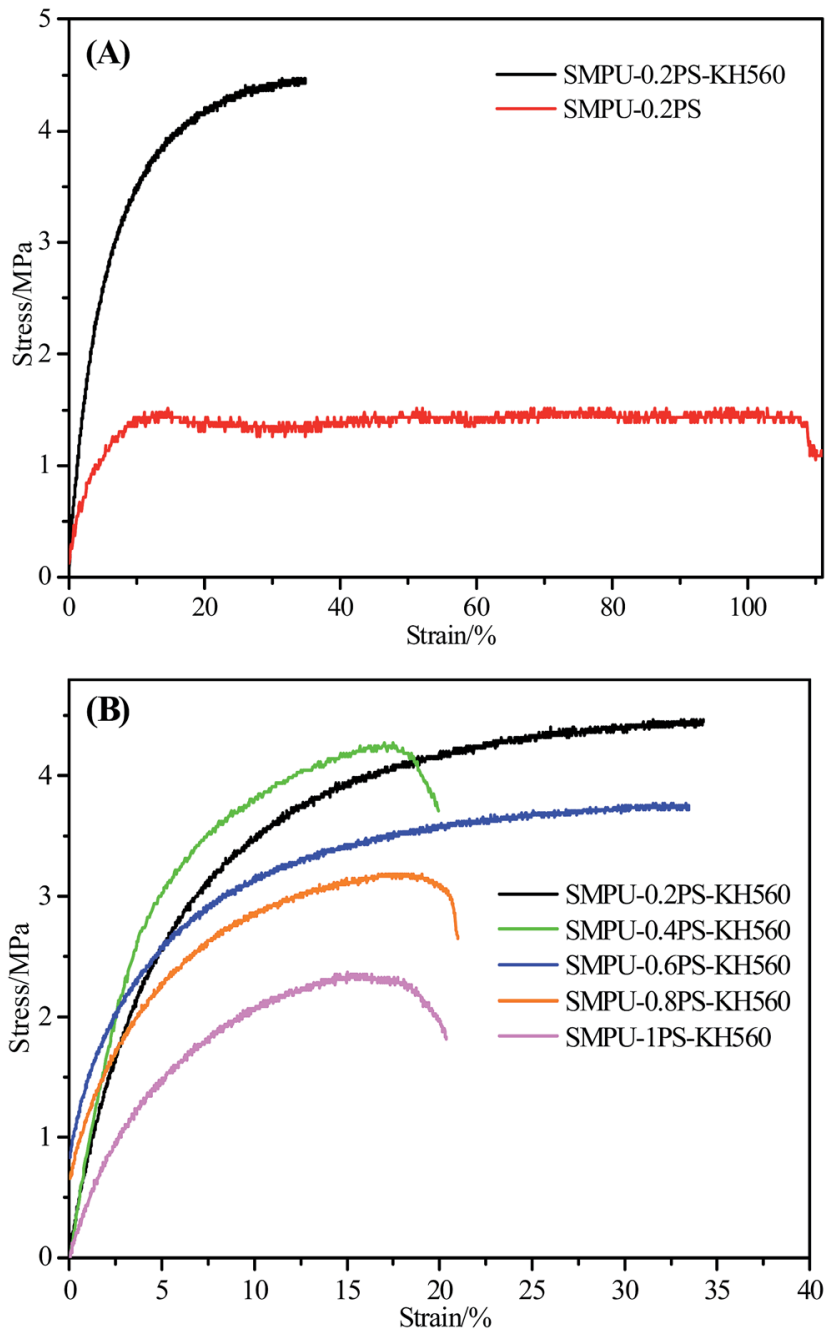

Fig. 7 Mechanical properties of ZSMPUS-KH560; ((A) for comparison of SMPU-0.2 PS and SMPU-0.2PS-KH560, (B) for comparison of ZSMPUs-KH560 with various zwitterionic segment contents).

decrease of $T_{\mathrm{g}}$ (see Fig. 8B). In comparison with the untreated ZSMPUs, the ZSMPUs-KH560 showed significantly higher glass modulus, semi-crystalline modulus, and rubber modulus (see Fig. 8C). The $T_{\mathrm{g}}$ of soft phase shifted to higher temperatures by incorporation of KH560 (see Fig. 8D). The plausible reason for the phenomenon could be that the incorporation of KH560 promoted the entanglement of particularly hard segments.

\subsection{Thermally induced shape memory properties}

The thermally induced shape memory properties of the target ZSMPUs-KH560 were analyzed by using a TA Instrument DMA Q200. Fig. 9A shows that the sample SMPU-0.4PS-KH560 could be deformed to nearly $100 \%$ strain when it was heated to $55^{\circ} \mathrm{C}$. After cooling below $20{ }^{\circ} \mathrm{C}$, more than $92.4 \%$ strain of SMPU0.4 PS-KH560 can be fixed. When SMPU-0.4PS-KH560 was reheated to $55{ }^{\circ} \mathrm{C}$, nearly $90 \%$ of the deformed strain was recovered. The other ZSMPUs-KH560 also showed this similar thermally induced shape memory behavior (see Fig. 9B). Overall, they had good shape fixity of above $94 \%$; however, the shape
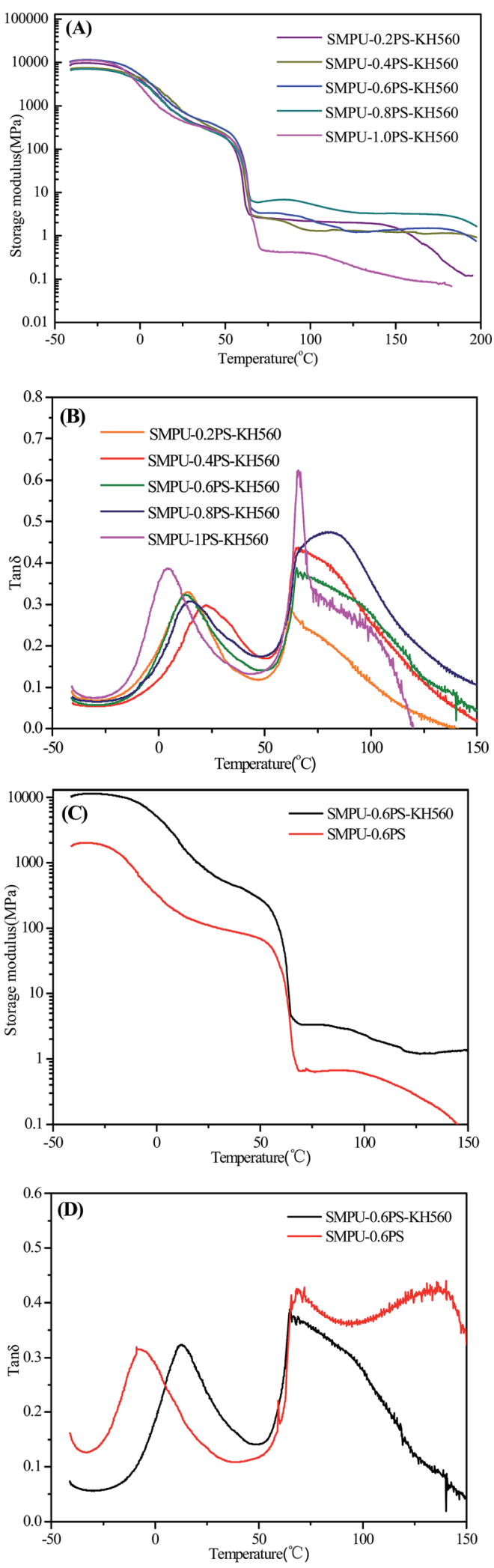

Fig. 8 The DMA curves (A, C - E', B, D - tan $\delta$ ) of ZSMPU-KH560 with various zwitterionic segments $(A, B)$ and a comparison of ZSMPUs and ZSMPU-KH560(C, D). 

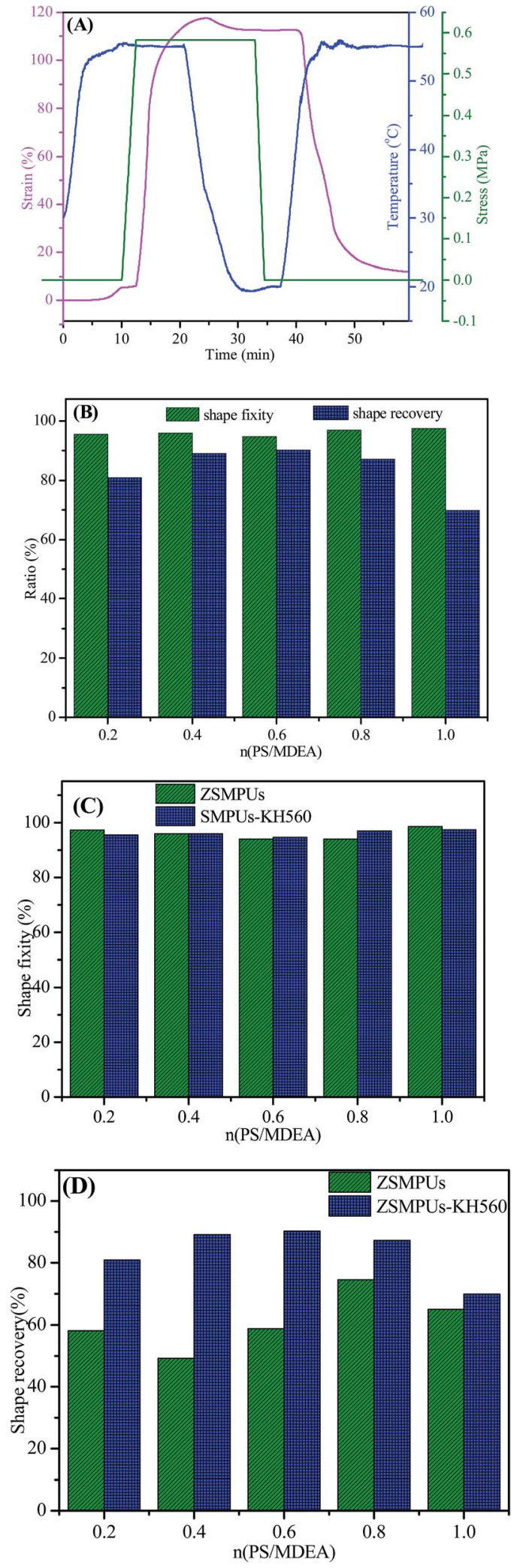

Fig. 9 Thermal-induced shape memory behaviors of ZSMPUs-KH560 (A) a typical shape memory curves of SMPU-0.4PS-KH560, (B) shape recovery and shape fixity of ZSMPUs-KH560 with various zwitterionic segment contents, (C) comparison for shape fixity of ZSMPUs and ZSMPUs-KH560, (D) comparison for shape recovery of ZSMPUs and ZSMPUs-KH560. recovery was quite different among ZSMPUs-KH560 with different zwitterionic segments. The shape recovery firstly increased with the increase in contents of zwitterionic segments, e.g. from $81.4 \%$ in sample SMPU-0.2PS-KH560 to $89.1 \%$ in sample SMPU-0.4PS-KH560 and to $90.5 \%$ in sample SMPU-0.6PS-KH560. Thereafter, the shape recovery decreased from $90.5 \%$ in sample SMPU-0.6PS-KH560, to $87.2 \%$ in sample SMPU-0.8PS-KH560 and to $69.5 \%$ in sample SMPU-1.0PSKH560. Hence, the shape recovery of ZSMPUs-KH560 can reach $90.5 \%$ by using silane coupling agent. In comparison with the untreated ZSMPUs, the ZSMPUs-KH560 not only have better shape fixity but also have a greater improvement in shape recovery (see Fig. 9C and D). The possible reason could be that the incorporation of KH560 produces side chains in the hard segments, which greatly promote the entanglement of hard segments, serving as physical netpoints for thermally induced shape memory properties.

\section{Conclusions}

The current research work provides a novel strategy to improve shape memory properties of ZSMPUs by using a silane coupling agent, KH560, via ring-opening reaction. The synergistic effects of zwitterionic segments and silane coupling agents on the ZSMPUs-KH560 were carefully investigated. The results demonstrate that the incorporation of KH560 does not affect the original molecular structure and morphology of ZSMPUs. The ZSMPUs-KH560 show hard phase-soft phase separation structure. The PEG soft segments display a good crystallizability, while the incorporation of KH560 promotes the entanglement of hard segments. Also, the silane coupling agent provides lubrication to influence the aggregation of soft segments, while the zwitterionic segments promote the formation of crystal seeds. Moreover, KH560 could compensate for the negative influences of zwitterionic segments, thereby providing better mechanical properties to ZSMPUs-KH560. Finally, shape memory tests certify that the semi-crystalline soft phase endows ZSMPUs-KH560 with good shape fixation of above $94 \%$ and the entangled hard domains significantly enhance the shape recovery of ZSMPUs to above $90 \%$. Thus, this kind of SMPU have good potential applications in smart biomedical fields.

\section{Conflicts of interest}

The authors declare that they have no conflict of interest.

\section{Acknowledgements}

The authors gratefully acknowledge the financial support from the Natural Science Foundation of China (Grant No. 51773120), Natural Science Foundation of Guangdong (Grant No. 2014A030313559 and 2016A030313050), the National Natural Science Foundation of Guangdong Province for Vertical Coordination Project (No. 201642), the Nanshan District Key Lab for Biopolymers and Safety Evaluation (No. KC2014ZDZJ0001A), the Science and Technology Project of Shenzhen City (Grant No. 
(Grant No. JCYJ20140828163633993, CYZZ20150827160341635, ZDSYS201507141105130, JCYJ20170412105034748).), the Guangdong Graduate Education Innovation Program for Postgraduate Demonstration Base of Joint Training, the Research Project of Shenzhen University (No. 201518), and the Top Talent Launch Scientific Research Projects of Shenzhen (827-000133).

\section{References}

1 B. Q. Y. Chan, Z. W. K. Low, S. J. W. Heng, S. Y. Chan, C. Owh and X. J. Loh, ACS Appl. Mater. Interfaces, 2016, 8, 10070-10087.

2 M. A. Kazemi-Lari, M. H. Malakooti and H. A. Sodano, Smart Mater. Struct., 2017, 26(5), 055003.

3 N. Zheng, J. J. Hou, Y. Xu, Z. Z. Fang, W. K. Zou, Q. Zhao and T. Xie, ACS Macro Lett., 2017, 6, 326-330.

4 J. Hu, Y. Zhu, H. Huang and J. Lu, Prog. Polym. Sci., 2012, 37, 1720-1763.

5 L. S. Zhang, S. S. Shams, Y. P. Wei, X. Q. Liu, S. Q. Ma, R. Y. Zhang and J. Zhu, J. Mater. Chem. A, 2014, 2, 2001020016.

6 M. D. Hager, S. Bode, C. Weber and U. S. Schubert, Prog. Polym. Sci., 2015, 49-50, 3-33.

7 E. Hornbogen, Adv. Funct. Mater., 2006, 8, 101-106.

8 W. Voit, T. Ware, R. R. Dasari, P. Smith, L. Danz, D. Simon, S. Barlow, S. R. Marder and K. Gall, Adv. Funct. Mater., 2010, 20, 162-171.

9 T. Xie, Nature, 2010, 464, 267-270.

10 S. Chen, H. Yuan, S. Chen, H. Yang, Z. Ge, H. Zhuo and J. Liu, J. Mater. Chem. A, 2014, 2, 10169-10181.

11 H. B. Lu, K. Yu, S. H. Sun, Y. J. Liu and J. S. Leng, Polym. Int., 2010, 59, 766-771.

12 T. Ohki, Q. Q. Ni, N. Ohsako and M. Iwamoto, Composites, Part A, 2004, 35, 1065-1073.

13 F. Cao and S. C. Jana, Polymer, 2007, 48, 3790-3800.

14 S. J. Chen, H. M. Yuan, Z. C. Ge, S. G. Chen, H. T. Zhuo and J. H. Liu, J. Mater. Chem. C, 2014, 2, 1041-1049.
15 M. K. Jang, A. Hartwig and B. K. Kim, J. Mater. Chem., 2009, 19, 1166-1172.

16 B. Zhou and Y. J. Liu, Int. J. Mod. Phys. B, 2009, 23, 12481253.

17 Q. H. Meng and J. L. Hu, Composites, Part A, 2009, 40, 16611672.

18 H. F. Jiu, H. Q. Jiao, L. X. Zhang, S. M. Zhang and Y. A. Zhao, J. Mater. Sci.: Mater. Electron., 2016, 27, 10720-10728.

19 J. T. Kim, B. K. Kim, E. Y. Kim, H. C. Park and H. M. Jeong, React. Funct. Polym., 2014, 74, 16-21.

20 C. Y. Bae, J. H. Park, E. Y. Kim, Y. S. Kang and B. K. Kim, J. Mater. Chem., 2011, 21, 11288-11295.

21 T. Hooshmand, R. van Noort and A. Keshvad, Dent. Mater., 2004, 20, 635-642.

22 X. X. Yan and G. Y. Xu, Prog. Org. Coat., 2012, 73, 232-238.

23 L. Bin, L. Song-Mei, L. Jian-Hua and Y. Mei, Appl. Surf. Sci., 2014, 315, 241-246.

24 D. Zhang, J. Wang, S. Wen, P. Wang, C. Yin and Z. Du, Adv. Mater. Phys. Chem., 2015, 5, 60-66.

25 P. J. Li, F. W. Nian, M. Zhang, M. M. Shen, Y. Q. Dai, H. Pang and B. Liao, J. Appl. Polym. Sci., 2016, 133.

26 S. J. Chen, Z. K. Mei, H. H. Ren, H. T. Zhuo, J. H. Liu and Z. C. Ge, Polym. Chem., 2016, 5773-5782.

27 S. J. Chen, F. N. Mo, Y. Yang, F. J. Stadler, S. G. Chen, H. P. Yang and Z. C. Ge, J. Mater. Chem. A, 2015, 3, 29242933.

28 S. J. Chen, F. N. Mo, F. J. Stadler, S. G. Chen, Z. C. Ge and H. T. Zhuo, J. Mater. Chem. B, 2015, 3, 6645-6655.

29 F. Mo, H. Ren, S. Chen and Z. Ge, Mater. Lett., 2014, 145, 174-176.

30 A. Magnaldo, M. Masson and R. Champion, Chem. Eng. Sci., 2007, 62, 766-774.

31 T. Wang, X. W. Wang, Y. C. Long, G. M. Liu and G. Z. Zhang, Langmuir, 2013, 29, 6588-6596. 